TTR

Traduction, terminologie, re?daction

\title{
Le bilinguisme officiel (français-anglais) au Cameroun : un problème d'aménagement efficace
}

\section{Dieudonné P. Aroga Bessong}

Volume 10, numéro 1, 1er semestre 1997

Langues, traduction et post-colonialisme

Languages, Translation and Post-Colonialism

URI : https://id.erudit.org/iderudit/037285ar

DOI : https://doi.org/10.7202/037285ar

Aller au sommaire du numéro

\section{Éditeur(s)}

Association canadienne de traductologie

ISSN

0835-8443 (imprimé)

1708-2188 (numérique)

Découvrir la revue

Citer cet article

Aroga Bessong, D. P. (1997). Le bilinguisme officiel (français-anglais) au Cameroun : un problème d'aménagement efficace. TTR, 10(1), 219-244. https://doi.org/10.7202/037285ar

\section{Résumé de l'article}

Le bilinguisme officiel (français-anglais) au Cameroun : un problème d'aménagement efficace - Cette étude vise à vérifier l'étendue de la prépondérance exclusive du français et de l'anglais dans la communication au sein de l'État camerounais, en raison de certaines contradictions (Renaud 1979 et 1987). Ces langues officielles sont censées y assurer les fonctions les plus hautes en présence de 236 langues autochtones. Ces dernières sont-elles réellement absentes des services publics? Quelles sont les attitudes des Camerounais envers elles et leurs communautés constitutionnelles respectives? Quelle serait une politique linguistique réaliste pour le Cameroun? Pour répondre à ces questions, nous avons, en 1993, soumis à 590 personnes dans les chefs-lieux administratifs des dix provinces du pays, un questionnaire fermé sur leurs choix de langue et les attentes linguistiques perçues par eux dans leurs contacts officiels. L'analyse des données a révélé l'existence d'une concurrence participative reflétant l'élargissement fonctionnel des langues non officielles. Une tension y est apparue entre le français et l'anglais, mais elle reflète les conflits politiques ambiants.

S'agissant des attitudes, le répondant devait porter son jugement sur les langues officielles et leurs communautés constitutionnelles respectives, par rapport à sept différenciateurs sémantiques inspirés de Gardner et Smythe (1976). L'analyse révèle : une haute appréciation des répondants de chaque communauté pour leur première langue officielle, l'évaluation négative de leur deuxième langue officielle par les anglophones, l'apparition des stéréotypes négatifs envers le pidgin, et la meilleure image que les anglophones auraient de leur communauté.

La concurrence participative entre langues officielles et non officielles, le nationalisme et la volonté d'ouverture des Camerounais, ainsi que leur aspiration à voir les hautes fonctions étendues aux langues locales, nous ont amené à proposer l'alphabétisation fonctionnelle en langues locales, pour la nécessaire valorisation des langues endogènes, dans un contexte minimisant la confrontation avec les langues officielles, et consacrant le passage du culturel à l'économique.

Tous droits réservés ( TTR: traduction, terminologie, rédaction — Les auteurs, Ce document est protégé par la loi sur le droit d'auteur. L’utilisation des 1997 services d'Érudit (y compris la reproduction) est assujettie à sa politique d'utilisation que vous pouvez consulter en ligne.

https://apropos.erudit.org/fr/usagers/politique-dutilisation/ 


\section{Le bilinguisme officiel (français-anglais) au Cameroun : un problème d'aménagement efficace ${ }^{1}$}

\section{Dieudonné P. Aroga Bessong}

\section{Problématique}

Les États plurilingues comme la République du Cameroun connaissent un important problème d'aménagement linguistique. Pour le résoudre, monolinguisme, bilinguisme exogène d'abord territorial, ensuite individuel, et plusieurs sortes de trilinguismes ont été tour à tour proposés (Ngijol, 1964, Fonlon, 1969, Towa, 1975, Tadadjeu, 1983, par exemple) sans donner entière satisfaction. Le bilinguisme officiel actuellement en vigueur aurait, selon Renaud (1987), abouti à une diglossie slangues officielles/langues nationales» qui est contraire à notre expérience de natif.

1 J'ai bénéficié d'une bourse du Gouvernement de la République du Cameroun pour cette recherche, effectuée en vue d'une thèse de doctorat en linguistique soutenue en septembre 1996 au département de linguistique et de traduction de l'Université de Montréal. Qu'il trouve ici l'expression de ma profonde gratitude. Toutes les erreurs qui pourraient être notées ne sont imputables qu'à moi. 
Langues de l'administration et de l'éducation héritées de la colonisation et censées assurer les fonctions les plus hautes en présence de 236 langues autochtones, le français et l'anglais monopolisent les ressources nationales en matière d'aménagement. Leur promotion quasi exclusive a-t-elle effectivement signé l'arrêt de mort des langues nationales (autochtones) ? En admettant que w[l]a langue est un élément important d'organisation sociale qui peut contribuer à l'amélioration des conditions de vie» (Djité, 1991, p. 122), la réponse à cette question essentielle devient fondamentale pour trouver une solution satisfaisante.

\section{Méthodologie}

Pour tenter d'y répondre, nous avons administré, de mars à juin 1993, à environ 330 élèves de la classe de première des établissements publics d'enseignement secondaire, ainsi qu'à 260 agents des secteurs public et privé du monde du travail, un questionnaire fermé sur les choix de langue des répondants et ceux qu'ils estiment être attendus de la part de leurs interlocuteurs, quand ils contactent, au bureau, des agents de l'État qui parlent : 1- la même première langue officielle (LO1) qu'eux, 2- leur seconde langue officielle (LO2), 3- le Pidgin English, 4- la/les même(s) langue(s) camerounaise(s) qu'eux. Car dans les contacts linguistiques entre Camerounais, la connaissance des langues que maîtrise l'interlocuteur est un paramètre important (Konig et al. 1983).

L'élimination des langues locales devrait se traduire, par exemple, par un faible choix des langues non officielles, même dans les contextes qui leur sont favorables, et surtout par une réduction de leur présence dans la pratique des élèves. De plus, la proportion des choix de ces langues devrait être nettement inférieure aux attentes des interlocuteurs, et davantage encore parmi les élèves et les jeunes en général. L'analyse de cette hypothèse tiendra compte de la sociolinguistique appliquée à des langues minoritaires comme le catalan (Boyer, 1991). Elle permettra de poser le problème de la dynamique d'évolution perceptible de cette diglossie (Baylon, 1991, Fishman, 1972).

Le deuxième volet du questionnaire porte sur les attitudes linguistiques. Le répondant devait indiquer s'il trouve chacune des langues officielles et le pidgin très, assez, moyennement, peu ou pas du tout riches, méprisables, compliqués, rémunerrateurs, instructifs, utiles ou 
importants. De même, il devait spécifier s'il estime les francophones et anglophones très, assez, moyennement, peu ou pas du tout paresseux, tribalistes, sérieux, méfiants, intelligents, polis ou honnêtes. Ce volet, inspiré de Gardner et Smythe (1976), devrait permettre de comprendre les résultats du premier et de les interpréter.

Notre enquête, qui tient compte notamment, de l'âge, du statut socioprofessionnel et du niveau d'étude des répondants, permettra de situer ces problèmes et d'élaborer une solution visant à inscrire le bilinguisme officiel au Cameroun dans la réalité ambiante dun plurilinguisme très dynamique. Notre approche intègre les résultats de l'analyse ethnolinguistique du multilinguisme camerounais effectuée par Dieu et Renaud (1983). Si l'aspect culturel a prédominé jusqu'ici, notre étude vise plutôt la valorisation économique des langues du terroir.

\section{Arrière-plan historique}

À l'issue de la Première Guerre mondiale, l'ancienne colonie allemande du Kamerun avait été divisée et confiée aux alliés victorieux par la Société des Nations : la Grande-Bretagne reçut le mandat d'administrer les Camerouns septentrional et méridional, et les rattacha à sa colonie du Nigéria. Dans le même cadre, la gestion du Cameroun oriental revint à la France. Transformés en tutelles après la création de l'ONU, ces mandats furent par la suite remis en cause par la forte aspiration des populations locales à l'indépendance. Le Cameroun français y accéda le $1^{\text {er }}$ janvier 1960.

Entre temps, poussées par le même désir d'émancipation, les populations du Cameroun méridional cherchèrent à retrouver les frères dont elles avaient été séparées. John Ngu Foncha, leur premier ministre à cette époque, qui rêvait de les voir accéder à l'indépendance, se heurta au refus de l'ONU pour qui la faiblesse démographique, et surtout économique, des Camerouns méridional et septentrional par rapport aux États indépendants voisins du Cameroun et du Nigéria, constituait un réel obstacle. Il n'avait d'autre choix que d'intégrer ses administrés à l'un ou l'autre de ces États, selon une formule dont il fallait convenir (Stark, 1980, p. 106).

Ahmadou Ahidjo, le jeune président de la République du Cameroun, opta pour un centralisme à moyen terme jouissant des faveurs 
des hommes politiques de son pays. Pratiquement entériné à la conférence constitutionnelle du 21 août 1961 à Foumban, le projet de constitution fédérale présenté par son gouvernement est devenu la constitution de la République fédérale du Cameroun proclamée le $1^{\text {or }}$ octobre 1961 et formée de l'ancienne République du Cameroun et du Cameroun méridional. Était ainsi instauré un bilinguisme officiel français-anglais paraissant placer sur un pied d'égalité ces deux entités alors que le Cameroun méridional ne représentait que le cinquième de la population totale. Le Cameroun septentrional avait choisi de rester avec le Nigéria.

\section{Aspects théoriques}

\subsection{Stabilité ou concurrence?}

\subsubsection{Diglossie}

Définissant la diglossie, Ferguson (1959, p. 336) en fait ressortir les deux critères essentiels : $1^{\circ}$ l'existence de deux variétés dialectales de la même langue : lune haute $(\mathrm{H})$, d'usage réservé aux interactions de type formel, l'autre basse (L), d'usage limité aux cadres d'intimité de la communication intra-groupe; $2^{\circ}$ ) la différence du statut de ces variétés dans le contexte social de leur utilisation.

Fishman (1967, p. 35) étend cette notion à la complémentarité de deux langues distinctes et pas forcément apparentées. De plus, il définit quatre types de situations possibles au niveau des rapports entre langues : ni diglossie ni bilinguisme, diglossie sans bilinguisme, diglossie et bilinguisme, bilinguisme sans diglossie. Pour Calvet (1987, p. 47), malgré cet élargissement, le concept de diglossie ne prevoit pas ala possibilité de ... diglossies enchâssées ... imbriquées les unes dans les autres». De plus, parce qu'elle s'attache à décrire des contextes stables, la typologie de Fishman ne suffirait pas pour caractériser le multilinguisme, souvent bien plus complexe.

Calvet propose d'analyser le phrilinguisme dans une perspective de pouvoir, tenant compte du rapport entre langue officielle et langue maternelle, Cette approche l'amène à retenir cinq types de plurilinguismes : à langue dominante unique, à langues dominantes 
minoritaires, à langue dominante minoritaire, à langues dominantes alternatives, à langues dominantes régionales.

D'après les sociolinguistes catalans, le castillan n'est utilisé comme langue unique que par une minorité (classe dominante ou fonctionnaires aétrangersw), mais il joue un rôle fonctionnel bien plus important (langue de l'administration et de l'enseignement). Pourtant son emploi n'a qu'une faible incidence au sein des populations. Ils voient là une diglossie, mais avec s'accent sur ... un déséquilibre sociolinguistique» (Boyer, 1991, p. 21) et non sur la stabilité. Elle sert de cadre d'analyse à un conflit linguistique entre langue dominante (usage officiel) et langue dominée (interactions d'intimité).

On voit tout l'attrait d'une telle optique pour la situation camerounaise, qui parâ̂t s'y prêter tout particulièrement. Baylon (1991, p. 150) note trois types d'évolution de la diglossie : le maintien de la situation, la convergence, dont le résultat est l'unification des variétés concernées (dialectes d'une même langue), ou l'élimination de l'une d'elles.

\subsubsection{Langue véhiculaire}

Observées du point de vue de leurs fonctions, les langues, selon Calvet (1987), se situent entre deux pôles : le grégaire, qui permet de limiter la communication (p. 79), et le véhiculaire, qui permet de marquer achu contraire sa volonté de rapprochement (p. 81). Parmi les principaux facteurs favorisant la véhicularisation des langues, Wardhaugh (1987, pp. 6-17) cite : les mouvements des populations; les villes, lieux privilégiés de contact, jouant ici un rôle capital; les conquêtes militaires, surtout depuis l'avènement de la colonisation; les prejugés répandus en faveur de certaines langues (français comme langue de culture en Afrique francophone); la religion, dont linfluence est souvent marquante sur le plan de l'orthographe; l'histoire, qui confere prestige et respect à certaines cultures et aux langues qu'elles utilisent; l'influence néo-coloniale, grâce au maintien par les élites des rapports linguistiques établis pendant la colonisation; l'attitude des locuteurs vis-à-vis des langues en contact. 


\subsection{Ethnicité et vitalité ethnolinguistique}

Fishman (1977) propose de distinguer trois dimensions de l'ethnicité : la paternité, dans le cadre de laquelle des liens biologiques entre les membres du groupe sont présumés; le patrimoine, regroupant les orientations et comportements transmis par les générations précédentes; la phénoménologie, liée à l'attitude de ces membres vis-à-vis de leurs liens avec leur groupe. Pour Yinger (1981), la puissance de l'ethnicité s'explique par trois séries de forces : celles découlant de la puissance permanente des liens primordiaux fondée sur la reconnaissance de rapports historiques d'interdépendance; celles issues de la prise de conscience de la réalité ethnique, dans un contexte de cohabitation sans obligation d'estimer ses partenaires; celles liées à l'utilisation de l'ethnicité dans la quête d'un mieux-être social et économique.

Trois catégories de facteurs essentiels rendent compte de la vitalité ethnolinguistique : dans la première figurent le degré d'estime dont jouit le groupe et le degré de confiance de ses membres en eux-mêmes, ainsi que le rôle de sa langue dans la société et les actions d'aménagement dont elle a été l'objet. Dans la deuxième se trouvent d'une part, la répartition géographique des membres du groupe, surtout compte tenu de son site ancestral d'implantation et de sa capacité à y maintenir son occupation exclusive, d'autre part, sa population et son taux de natalité. La dernière concerne l'appui informel (groupe de pression) ou formel (présence de sa langue dans divers médias ou institutions) dont il jouit.

\subsection{Politique linguistique}

Normalement, toute action d'aménagement, pour minimiser le risque d'échec, doit reposer sur deux fondements : $1^{\circ}$ la situation linguistique de la région où l'action est prévue (évaluation de l'état de la description des langues en présence); $2^{\circ}$ la situation démolinguistique et sociolinguistique (statistiques sur ces langues, leur statut, leur distribution sociale, et les actions menées en vue d'une meilleure utilisation).

Eu égard a la procédure, notre proposition se limite à l'aménagement du wstatut". Initialement considéré comme uthe recognition by a national government of the importance or position of one language in relation to others $\nsim($ Cooper, 1989, p. 32), ce terme s'applique désormais 
également à l'affectation de fonctions précises à une langue donnée. Appel et Muysken (1992, p. 56) relèvent quatre facteurs pouvant l'influencer : les facteurs socio-démographiques (nombre de langues et de leurs locuteurs ainsi que leur répartition géographique); les facteurs linguistiques, notamment le statut des langues en présence, les similitudes ou dissemblances entre elles et leur état d'élaboration; les facteurs socio-psychologiques, qui concernent tout le problème des attitudes linguistiques; les facteurs religieux, liés aux relations entre langue et religion.

\section{Résultats}

\subsection{Choix et attentes linguistiques}

Les données pour l'ensemble du pays font ressortir une situation apparemment stable pour les langues officielles. Dans le scénario général, près d'un tiers des choix de langue iraient à l'anglais et les deux autres tiers au français, à l'écrit comme à l'oral. Ce rapport est assez proche de I'équilibre (moitié-moitié) lorsque les répondants contactent un agent parlant leur seconde langue officielte (anglais pour les francophones, français pour les anglophones), toujours sans distinction de mode de contact. Il faut souligner la très faible proportion des choix de langues non officielles dans ces contextes : elles plafonnent a $7 \%$ (taux composé). La situation d'ensemble est très similaire en ce qui concerne la perception des attentes.

Par contre, en présence d'agents pidginophones ou parlant la/les même(s) langue(s) camerounaise(s) que les répondants, ces deux catégories de langues seraient choisies pratiquement dans les mêmes proportions à l'écrit ( $12 \%$ et $11 \%$ respectivement). Cela peut paraître faible; mais quand on sait que le pidgin et ces langues sont réputés non écrits et sont, en principe, exclus du domaine public, uniquement réservé aux langues officielles, l'observation prend toute son importance. À toutes fins pratiques, ces taux tripleraient, à l'oral, dépassant parfois le choix de la seconde langue officielle, logiquement privilégiée en raison du domaine. En ce qui concerne la perception des attentes, le taux face aux pidginophones atteint $28 \%$, et $18 \%$ face aux locuteurs de même(s) langue(s) camerounaise(s). À loral, ces taux doubleraient pour les deux catégories de langues par rapport aux choix. 
S'agissant de la comparaison des deux communautés et d'après les indications de la figure 1 ci-dessous, pour le scénario général les francophones choisissent le français à $90 \%$ au moins, les anglophones optant pour l'anglais à $80 \%$ au moins, à l'écrit et à l'oral. Dans les deux groupes le choix de la LO1 dépasse les attentes. Généralement assez bas, celui de la LO2 (anglais pour les francophones, français pour les anglophones) est en deçà des attentes. Face à un interlocuteur parlant leur LO2, ces choix, à l'écrit et l'oral, se situent entre $40 \%$ et $50 \%$ pour les deux communautés. Mais ils sont bien en deça des attentes.

Figure 1 : Choix et attentes linguistiques dans les deux communautés

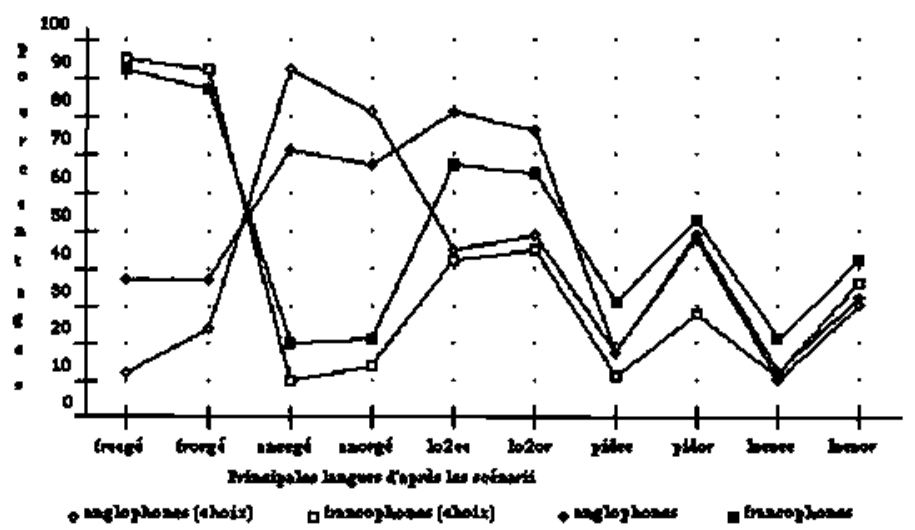

Frecgé = français écrit : scénario général; frorgé = français oral : scénario général; anecgé = anglais écrit : scénario général; anorgé = anglais oral : scénario général; lo2ec = deuxième langue officielle : écrit; lo2or = deuxième langue officielle : oral; pidec = pidgin écrit; pidor = pidgin oral; lacaec = langues camerounaises : écrit; lacaor $=$ langues camerounaises : oral.

En présence d'un interlocuteur pidginophone, les choix des anglophones correspondent à peu près aux attentes tant à l'écrit (17\%) qu'à l'oral (45\%). La situation est similaire en présence d'un interlocuteur parlant la/les même(s) langue(s) locale(s) qu'eux. Face au pidginophone, les choix des francophones sont à la fois inférieurs à ceux des anglophones et à peu près de moitié inférieurs aux attentes. Face aux locuteurs de langues camerounaises communes, leur choix par les francophones 
correspondent, à toutes fins pratiques, à ceux des anglophones, mais restent inférieurs aux attentes.

L'apparente faiblesse, déjà notée, des taux pour ces langues non officielles à l'écrit se retrouve ici, mais son importance a été expliquée. Les proportions obtenues pour ces langues par certaines catégories de la population sont plus grandes. Ainsi, le pidgin est choisi à $22 \%$ et présumé attendu à $47 \%$ à l'écrit par les lycéens bilingues francophones, les taux respectifs pour l'oral atteignant $57 \%$ et $82 \%$. Les langues camerounaises, quant à elles, sont choisies à $16 \%$ et présumées attendues à $26 \%$ à l'écrit par les éleves francophones, les proportions respectives pour l'oral étant de $35 \%$ et $48 \%$. Dans ces conditions, les langues non officielles se voient offrir un espace considérable d'utilisation.

Les marges entre attentes et choix effectifs peuvent sembler assez importantes, surtout chez les francophones, pour appuyer l'hypothèse de la disparition progressive de ces langues. Ce serait ignorer l'influence sur les choix et attentes du domaine (les services publics), de la limitation géographique de l'espace pidginophone en milieu francophone (quatre des dix provinces), des tabous liés à la pratique de ces langues, et de l'exagération de la supériorité culturelle du français, entretenue par l'élite francophone.

Lafontaine souligne : «... en interrogeant les enfants à l'école, on ne se trouve évidemment pas dans des conditions fovorables pour voir émerger des attitudes positives vis-à-vis d'une variété qui est rejetée de ce contextè (1986, p. 69). Puisque les élèves, qui constituent la majorité de notre échantillon, ont été interviewés en milieu scolaire, les taux de choix ou de perception des attentes pour les langues non officielles auraient été négativement influencés par ces conditions. $\mathrm{La}$ réalité de ce champ appréciable d'expression des langues non officielles dans les services publics serait donc bien plus importante. Généralement masqué par le domaine dans lequel les contacts au bureau sont effectués, cet espace se découvre lorsque les occasions - et elles sont nombreuses - s'y prêtent.

La relative importance des pourcentages des choix et attentes perçues du pidgin par les jeunes et les élèves ne semble pas correspondre à la perception onégative de cette langue que refletent les résultats de leurs attitudes envers elle. Le contexte d'administration du questionnaire 
semble donc les avoir amenés à exprimer le rejet, déjả mentionné, de cette langue du milieu scolaire.

\subsection{Attitudes linguistiques}

\subsubsection{Langues officielles}

Les répondants - surtout anglophones - ont une perception très positive de leur LO1 : les marges pour chaque différenciateur, entre les taux pour le français et l'anglais, sont appréciables dans la perception des anglophones. De plus, elles sont favorables a l'anglais, pour n'importe laquelle des six variables choisies, toutes catégories confondues. Par contre, elles le sont souvent moins, principalement pour les différenciateurs à caractère économique, chez les francophones, indépendamment de la variable et des catégories en cause. Par ailleurs, les anglophones auraient une perception moins positive de leur LO2 que les francophones.

Figure 2 : Différenciateurs liés au statut

\begin{tabular}{|c|c|c|c|c|c|}
\hline $\min _{x \rightarrow 3}$ & Fofferein- & $\begin{array}{l}\text { Rémmingra- } \\
\text { teur (95) }\end{array}$ & Chi 2 & $\begin{array}{l}\text { Instruc- } \\
\text { tif }(9)\end{array}$ & Ohi 2 \\
\hline \multirow{2}{*}{$\begin{array}{l}\mathbf{A} \\
\mathbf{n} \\
\mathbf{g} \\
\mathbf{1} \\
\mathbf{a} \\
\mathbf{1} \\
\mathbf{3}\end{array}$} & $\begin{array}{l}\text { Anglo- } \\
\text { phones }\end{array}$ & 84 a 100 & \multirow{2}{*}{$\left|\begin{array}{l}p \\
\mathbf{c} \\
0,05\end{array}\right|$} & 91 à 100 & \multirow{2}{*}{$\mid \begin{array}{l}\frac{p}{3} \\
0,05\end{array}$} \\
\hline & $\begin{array}{l}\text { Franco- } \\
\text { phones }\end{array}$ & $50 \times 75$ & & $85 \div 94$ & \\
\hline \multirow{2}{*}{$\begin{array}{l}\mathbf{F} \\
\mathbf{r} \\
\mathbf{n} \\
\mathbf{n} \\
\mathbf{f} \\
\mathbf{a} \\
\mathbf{i}\end{array}$} & $\begin{array}{l}\text { Anglo- } \\
\text { phones }\end{array}$ & 66 à 86 & \multirow{2}{*}{$\left|\begin{array}{l}p \\
3 \\
0,05\end{array}\right|$} & 6787 & \multirow{2}{*}{$\mid \begin{array}{l}p \\
0,05\end{array}$} \\
\hline & $\begin{array}{l}\text { Franco- } \\
\text { phones }\end{array}$ & $51 \div 67$ & & 92 में 96 & \\
\hline
\end{tabular}


Une observation intéressante porte sur certains différenciateurs liés au statut. Ainsi, 84 à $100 \%$ des anglophones estiment l'anglais rémunérateur, mais 66 à $86 \%$ trouvent le français rémunérateur (voir figure 2). Ces forts taux indiqueraient l'importance des LO, de la question linguistique et des frustrations liées au fait de maîtriser une langue peu valorisée alors qu'on souhaiterait se faire une place au soleil. Par contre, 67 a $87 \%$ des anglophones disent le français instructif, mais 91 à $100 \%$ l'affirment pour l'anglais (voir figure 2). L'observation pour le français constitue probablement un indice de l'insatisfaction des anglophones, pour qui cette langue serait un obstacle à leur mobilité sociale, d'où la frustration déjà évoquée. Quant aux pourcentages très élevés obtenus pour l'anglais dans les deux cas, ils confirmeraient qu'il a acquis valeur de ralliement pour sa communauté constitutionnelle (Hamers et Blanc, 1992).

\subsubsection{Le Pidgin English}

Quant au pidgin, alors que la majorité des catégories de francophones l'estiment non important (40-59\%), la moitié des classes d'anglophones sont du même avis. La qualification de cette langue comme non instructive obtient les plus forts taux (70-84 \%) en raison de son bannissement des milieux scolaires. Toutefois elle est jugée non méprisable et utile dans des proportions intéressantes (55-67\% pour les anglophones, $42-64 \%$ pour les francophones).

\subsubsection{Les communautés}

Les anglophones minoritaires semblent avoir une perception à la fois plus positive et plus claire de leur communauté que les francophones : ses membres ne seraient ni paresseux, ni tribalistes, ni méfiants, alors que les francophones, jugés non paresseux en proportion moins importante, sont dits tribalistes et méfiants, toutes variables et catégories confondues (voir figure 3). Les anglophones estiment aussi que les ressortissants de leur communauté sont sérieux, polis et honnêtes, contrairement à ceux de la communauté francophone.

Pour leur part, tout en disant que les anglophones ne sont pas paresseux, les francophones ont des opinions très partagées quant à leur appréciation des membres de leur propre communauté sur ce point; ils sont également partagés quant à la propension au tribalisme des 
ressortissants des deux groupes, qu'ils jugent par ailleurs méfiants. Les anglophones seraient plus sérieux (comparativement à une opinion sur les francophones en général très partagée) et bien plus polis que les francophones trouvés moins honnêtes que les anglophones (voir figure 3). Ces observations sont valables sans distinction de variable ou de catégorie.

Figure 3 : Perception des communautés

\begin{tabular}{|c|c|c|c|c|c|c|c|c|}
\hline $\begin{array}{l}\text { Pi } \\
\text { Com } \\
\text { mun }\end{array}$ & 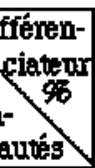 & $\begin{array}{l}\text { pares- } \\
\text { seux }\end{array}$ & $\begin{array}{l}\text { trba- } \\
\text { listes }\end{array}$ & sérieux & méfiants & $\begin{array}{l}\text { intent- } \\
\text { gents }\end{array}$ & polis & houne- \\
\hline \begin{tabular}{|l|} 
A \\
n \\
glo
\end{tabular} & \begin{tabular}{|l|} 
arglo \\
pho \\
nos
\end{tabular} & 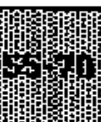 & ${ }^{4}$ & $60-93$ & ${ }^{\prime}$ & & 60-93 & $60-93$ \\
\hline $\begin{array}{l}\text { pu } \\
\text { nes }\end{array}$ & $\begin{array}{l}\text { frame } \\
\text { qpho } \\
\text { nes }\end{array}$ & 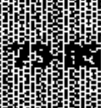 & & 41-70 & & $?$ & 65-84 & infér \\
\hline $\begin{array}{l}\mathrm{Fr} \\
\text { an } \\
\mathrm{co}\end{array}$ & \begin{tabular}{|l|} 
englo \\
pho \\
mes
\end{tabular} & 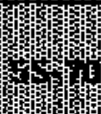 & $45-60$ & 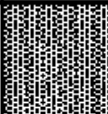 & $45-60$ & $\begin{array}{l}\text { inf. de } \\
\text { mnitts }\end{array}$ & & \\
\hline on & \begin{tabular}{|l|} 
framc \\
opho \\
ness
\end{tabular} & & & 39 & & $?$ & $40-49$ & \\
\hline
\end{tabular}

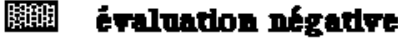 \\ $\square$ évaluation positive \\ [i:日: éralnution partagée}

Les attitudes des anglophones et francophones envers les anglophones se distinguent d'après tous les différenciateurs excepté la richesse. Elles paraissent moins diverger à propos des francophones puisque seules la propension au tribalisme, l'intelligence et la politesse, différenciateurs déjà essentiels chez les anglophones, sont concernées. 


\section{Discussion}

\subsection{Stabilité ou concurrence : quelle orientation ?}

S'agissant de la diglossie langues officielles/langues nationales, les résultats qui précèdent n'indiquent pas une situation de cloisonnement des fonctions entre ces langues. Les non-officielles sont résolument présentes dans le domaine de prédilection des officielles. Déjà, certaines nonofficielles, y compris le pidgin, sont utilisées à l'intérieur du cadre religieux pour la liturgie, l'enseignement ecclésial et la traduction de livres sacrés comme la Bible. Plusieurs sont présentes même dans les tribunaux (traduction consécutive des plaidoiries), et sont utilisées dans des programmes de radiodiffusion limitée (une quarantaine pour les dix stations radiophoniques provinciales).

La présence des langues non officielles dans les services publics paraît donc légitimée par ces éléments, de portée parfois symbolique, caractéristiques des variétés $H$ (hautes) en contexte de diglossie. Par ailleurs, le français aurait donné naissance à une variété "courante» (Manessy et al. 1992), dont l'usage (relativement peu étendu) s'apparenterait à celui du Pidgin English. Toutefois, le pidgin et ce français courant semblent essentiellement exclus du cadre des communications intra-groupe, caractéristiques des variétés $\mathrm{L}$ (basses).

Le concept de diglossie - même celui incluant des enchâssements, proposé par Calvet (1987) - paraît donc inapproprié pour saisir cette complexité évolutive de la réalité linguistique du Cameroun. L'analyse du rapport fonctionnel entre ces catégories de langues sous l'angle d'un conflit entre les dominantes et les dominées ne peut être retenue ici que pour traduire la perspective des décideurs. Les choix des usagers tendent plutôt à indiquer une situation de concurrence marquant la volonté de participation des variétés exclues, en même temps que l'élargissement fonctionnel de leurs concurrentes. Cette vision correspond à la culture camerounaise, qui encourage la collaboration (Todd, 1983).

La forte vitalité des langues se traduit donc, pour les officielles et surtout le français, par leur transformation en lingua franca et, à défaut, en variété superposée. Celle des non-officielles aboutit à un élargissement fonctionnel, qui constitue une stratégie de maintien qu'une action humaine 
délibérée contribuerait à consolider. La vitalité des langues non officielles se fonde également sur le nationalisme des Camerounais, signe de leur confiance en eux-mêmes, et sur le maintien d'une occupation exclusive, par leurs locuteurs, de leurs sites ancestraux d'implantation, peu perturbés par les mouvements migratoires vers les villes.

Le point de non-retour ne serait donc pas encore atteint, du point de vue des usagers, pour réaliser le monolinguisme implicite dans la politique aussi bien que dans certaines analyses. Mais pour briser la quadrature du cercle, les options d'aménagement devraient, comme en Catalogne, viser le soutien des langues non officielles alors qu'elles sont encore largement utilisées.

Chaque catégorie de langue cherche à s'ouvrir des espaces fonctionnels nouveaux, mais pas nécessairement au détriment de l'autre. Cette recherche prend la forme d'un élargissement concurrentiel, mais non exclusif, de l'espace fonctionnel des langues locales en raison de leur forte vitalité. Le phénomène traduit l'ouverture des jeunes aux nouveaux rôles ainsi acquis et perçus comme un enrichissement. La promotion officielle de langues exogènes dans un contexte sans réelle domination parmi la multitude de langues minoritaires pourrait donc aboutir à un enrichissement, et non pas à la seule polarisation fonctionnelie en termes de gains ou de pertes.

L'hypothèse d'un changement simplement inéluctable, sous-jacente à l'introduction, est donc à rejeter, puisque les jeunes, tout comme les élèves, manifestent une réelle disponibilité à utiliser les langues non officielles ou à estimer souhaitable leur utilisation dans un cadre qui leur est fort peu propice.

\subsection{Langue et identité}

Les indices de tension sur le plan des langues officielles ne manquent pas. Leurs choix sont parallèles entre les deux communautés, chacune accordant priorité quasi absolue à sa première langue officielle dans le contact général à l'écrit. Cela correspond à la perception très positive que les répondants ont de leur première langue officielle. De plus, moins la première est attendue, plus elle est choisie ; c'est l'inverse pour la seconde. 
Et cela est valable pour les deux communautés. À quoi seraient dues ces tensions?

Dans la communauté anglophone, le patrimoine, surtout ses éléments acquis grâce au système éducatif ou liés à d'autres aspects de la culture anglaise, comme diverses formes d'organisation et de gestion sociales, devient important, tout comme la phénoménologie. La langue anglaise, symbole de cette culture à protéger, est érigée en instrument de revendication politique, et sert de levier dans le pays pour avoir gain de cause dans toutes sortes de luttes. Cela explique que les anglophones aient une perception plus positive de leur première langue officielle que les francophones, et que leur attitude envers leur seconde langue officielle soit moins positive que celle des francophones envers l'anglais (Hamers et Blanc, 1992).

Contrairement au pronostic en leur défaveur d'après les résultats d'études antérieures, les anglophones, minoritaires, semblent avoir une perception a la fois plus positive et plus claire des membres de leur propre communauté que les francophones. En devenant une valeur identitaire centrale dans la communauté anglophone, l'anglais contribue donc au renforcement du statut du groupe ainsi manifesté, et apparemment reconnu par la communauté francophone.

L'absence de consensus entre les deux communautés sur la gestion publique aurait fragilisé la consolidation de valeurs partagées dans l'ensemble du pays. Le contexte politique national, ressenti comme coercitif par la communauté anglophone minoritaire, amène naturellement cette dernière à prendre conscience des possibilités que lui offre l'ethnicité et à remettre en question les valeurs sociales nationales d'intégration préconisées par le pouvoir en place. Les tensions notées plus haut dans les choix et attentes linguistiques entre les membres des deux communautés sont donc le reflet de cette insatisfaction politique, perceptible au niveau de l'évaluation du français, obstacle à la mobilité des anglophones.

Ces tensions dépassent le cadre de la présente étude. Des gages politiques sont nécessaires pour assurer le maintien, voire le respect, de la culture des partenaires. Dans ce sens, une politique linguistique plus explicite paraît indispensable. Mais au-delà, il importe de restaurer la 
confiance en recherchant un réel consensus (Hamers et Blanc 1992, p. 117).

\section{Politique linguistique}

\subsection{Perspective}

Dès son origine, la politique linguistique mise en ceuvre au Cameroun fut đominée par des préoccupations éducatives. Quatre facteurs permettent de comprendre son évolution pendant la période coloniale :

1) les administrations coloniales favorisaient généralement l'utilisation de la langue de la métropole (Stumpf 1979);

2) les associations missionnaires religieuses préconisaient en priorité l'utilisation des langues locales, souvent sur la base de principes évangéliques. (Toutefois, leur propre propension à la concurrence encouragea les administrations coloniales à s'en faire des alliés);

3) la culture européenne fascinait les populations locales; la maîtrise de langues exogènes devint une de leurs demandes importantes (Stumpf 1979, p. 16);

4) des considérations politiques nouvelles intervenaient : tendances unificatrices et centralisatrices, européocentrisme linguistique et récompense des collaborateurs au détriment des autonomistes.

L'ONU, organisme mandant au Cameroun, était favorable à la promotion des langues locales les plus parlées (Stumpf, 1979, p. 103). Mais à l'indépendance, le $1^{\text {er }}$ janvier 1960 , le français est demeuré la langue officielle. Après la proclamation de la République fédérale du Cameroun le $1^{\text {er }}$ octobre 1961, le bilinguisme officiel (anglais-français) fut institué. Puis, on est passé de la promotion du bilinguisme dans les territoires ex-coloniaux, à celui des individus (voir Fonlon, 1975, et Biya, 1987).

Le vou exprimé en 1961 de voir les deux langues «importées» faire place un jour à mune langue et une culture autochtones "Benjamin 1972, p. 124) fut repris par Ngijol en 1964, mais sans succès, par crainte que la langue du chef de l'État de l'époque ne soit imposée. D'autres proposeront l'institution de langues régionales, intégrant parfois cette option dans le cadre du trilinguisme extensif. Selon Tadadjeu (1985, p. 2), chacune des 236 unités-langues entre lesquelles l'intercompréhension est 
impossiblex doit être utilisée dans son propre cadre, dans une perspective fonctionnelle.

Cette insistance sur l'absence d'intercompréhension entre les langues nationales semble ne pas tenir compte des travaux de Dieu et Renaud, qui ont identifié neuf aires d'intercompréhension, et elle ignore également la vêhicularisation de certaines langues signalée par Kœnig et al. (1983). En revanche, dans le cadre d'une approche historicostructurelle tenant compte des motivations des choix individuels et collectifs, Mopoho (1993) suggère le modèle de partenariat. L'essentiel des propositions faites à ce jour se cantonne à l'introduction des langues nationales dans le système scolaire classique, conformément au rapport de IUNESCO (1953) sur l'utilisation des langues vernaculaires dans l'éducation.

\subsection{Critique}

Avec la résolution politique en 1985 d'encourager de développement des langues nationales, facteurs déterminants de notre identité culturelle ...» (Tadadjeu 1987, p. 9), une certaine complémentarité entre ces langues et les langues officielles semblait reconnue. Toutefois, demeurée symbolique, elle n'a rien changé au paysage sociolinguistique camerounais.

Les résultats de notre analyse ont permis de constater que la situation ne relève pas d'une simple complémentarité. Une certaine concurrence existerait entre les deux groupes de langues. Leur vitalité les porterait à s'ouvrir des espaces fonctionnels nouveaux. Il s'agit simplement d'en tenir compte. Toutefois, les propositions d'aménagement du statut des langues non officielles en terme d'affectation fonctionnelle liée à l'éducation, principal domaine de promotion des langues officielles, ont plutôt exacerbé la menace de les remplacer.

Dans les orientations publiques, le passage de la perspective territoriale à l'action individuelle, du moins en ce qui concerne le bilinguisme officiel, semble avoir focalisé la recherche de solutions aux questions linguistiques à ce même niveau. On se bornait à savoir combien de langues le citoyen moyen peut ou doit parler. Or cette approche a l'inconvénient d'être porteuse d'insécurité. Pour avoir quelque chance 
d'aboutir, toute proposition d'aménagement devrait la minimiser et en réduire l'effet redouté de confrontation (Valdman, 1979).

\section{3. Éléments pour un aménagement réaliste}

Les bases sociolinguistiques généralement admises de l'aménagement linguistique sont regroupées, pour ce qui concerne le Cameroun, dans les ouvrages de Dieu et Renaud (1983) et Koenig et al. (1983). Le consensus obtenu en 1985 reconnaît aux particularismes (notamment linguistiques) sun fonds naturel à faire valoir (Gaillard 1989, p. 117). Sur la base de ces données, il est possible de proposer un modèle réaliste d'aménagement tenant compte de la concurrence participative entre langues officielles et non officielles qu'indiquent les résultats de notre enquête.

Compte tenu de l'histoire linguistique camerounaise, il est permis de penser que toute valorisation subite et importante, qui n'officialiserait pas les aspirations linguistiques ou le vécu sociolinguistique des populations, serait vouée à l'échec. Ces aspirations linguistiques furent à l'origine de l'introduction de l'anglais dans l'enseignement chez les anglophones; quant au vécu sociolinguistique, il justifie en partie l'intérêt persistant des Camerounais pour le pidgin, malgré tous les efforts successifs des administrations européennes et autochtones visant à l'enrayer.

\subsection{Notre proposition}

\subsubsection{Orientation d'ensemble}

La concurrence non exclusive identifiée entre langues officielles et non officielles, pour faire l'objet d'une politique consensuelle, doit contribuer à dissiper les appréhensions existantes par rapport aux langues officielles, tout en autorisant une valorisation des non officielles ne reposant pas uniquement sur l'affectation fonctionnelle. Cette valorisation doit pouvoir aider à la revitalisation économique, un des principaux éléments de réussite du maintien de langues minoritaires comme le catalan (Tabouret-Keller 1992, p. 273), qui est absent du contexte camerounais. L'alphabétisation fonctionnelle en langues locales, à l'intention d'une clientèle non scolaire au départ, apparaît comme une solution réaliste et productive. 
Dans cette optique, les langues officielles continueraient d'être utilisées dans le système formel d'éducation. Pour apaiser les tensions notées en ce qui concerne les langues officielles, l'orientation des programmes en vue de l'appui au groupe minoritaire, grâce au renforcement de sa langue et de sa culture, paraîtrait souhaitable. L'introduction de l'anglais renforcé comme option (parallèlement à l'espagnol ou l'allemand) à certains examens officiels va dans ce sens. La mesure pourrait être généralisée. Au-delà des gages plus généraux à donner, on pourrait aussi mettre fin à l'apparente duperie au niveau de l'application des initiatives linguistiques touchant les langues officielles, et faire preuve de respect et de transparence, notamment en ce qui a trait au contrôle des examens officiels, ce qui semble en bonne voie.

Quant aux langues non officielles, la démarche la plus appropriée consisterait à partir de la périphérie du système éducatif actuel en se concentrant sur les exclus de ce système, $\mathrm{y}$ compris les populations non alphabétisées, grâce à l'alphabétisation fonctionnelle, car relle peut être un catalyseur de mobilisation sociale et de changement des comportements et des mentalités au niveau social, économique et psychologique» (Djité 1991, p. 132). Cette démarche ne serait pas une nouveauté au Cameroun, où elle a été instituée en 1964, en langues officielles toutefois. Constaté déjà en 1978 en raison de l'extrême réduction des effectifs, l'échec de cette démarche pourrait être renversé par l'utilisation des langues locales si elles sont valorisées pour permettre de satisfaire les aspirations des populations (Todd 1983, p. 168).

\subsubsection{Ethnicité et développement}

Plusieurs ont prétendu qu'une telle mise en valeur de langues locales pourrait encourager les tensions ethniques (Valdman, 1979; Tadadjeu, 1988); largument est souvent exagéré. Des langues ont été choisies pour l'éducation (avant l'indépendance) et pour la radiodiffusion (après l'indépendance) sans que cela provoque une grave crise sociale. Il y a lieu de tirer profit des éléments dynamiques de l'ethnicité, pour la mettre au service du développement.

À la faveur du retour au pluralisme politique, on assiste à la résurgence d'associations à caractère ethnique, comme le Ngondo, et à la constitution de groupes de pression tels que l'Essingan ou le Laakam 
rassemblant des ressortissants de populations qui se réclament d'une identité ethnique commune. En leur sein, une ou quelques langues ont été véhicularisées, voire promues par les missionnaires. Cet acquis est exploitable dans le cadre d'orientations claires, pouvant être définies dans une charte appropriée. Ces organismes où les statuts suivent les lignes de force des rapprochements et ressemblances signalés, au plan sociolinguistique, par Dieu et al. (1983), ouvrent des espaces nouveaux de cohésion moins primaire, susceptibles de servir de base aux revendications d'un mieux-être ethnique au sens susvisé. À notre avis, un aménagement réaliste doit tenir compte de cette donne, et viser à moyen terme l'affectation de fonctions nouvelles à quelques-unes des variétés ainsi véhicularisées.

Grâce à l'alphabétisation fonctionnelle en langues locales, dont la clientèle serait, au départ, essentiellement non scolarisée, s'instaurerait un partage de rôles sans réelle menace pour les langues officielles et offrant cette reconnaissance explicite et souhaitée des langues non officielles, reconnaissance susceptible de consolider leur valorisation. Notre proposition ne vise donc pas à substituer les langues nationales aux langues officielles.

L'aménagement linguistique ainsi conçu contribuerait à restaurer la confiance tout en comblant des besoins économiques et culturels. Il ôterait les barrières administratives et psychologiques entravant les échanges entre l'État et les citoyens et permettrait de résoudre le déficit de communication entre eux.

L'affectation de nouvelles fonctions dans ce cadre offre des bénéfices économiques et culturels d'une instrumentation modérée, assise sur la valeur que les Camerounais attachent aux langues locales et que reconnaissent la majorité des études qui s'y rapportent. Le cadre linguistique semble convenable, compte tenu des aires de véhicularité indiquées. L'équilibre viendrait de cette même modération, ainsi que du respect des tendances ressortant de l'étude du comportement linguistique des populations. L'usage en cours des langues locales dans le domaine religieux et radiophonique devrait faciliter l'acceptation de cette option.

Le cadre que nous proposons paraît propice à une mise en ceuvre réaliste du modèle de partenariat entre le pouvoir et les collectivités 
locales proposé par Mopoho (1993). Une charte des langues ferait partie de l'appareillage réglementaire mis en place par le premier. Elle permettrait une contribution plus sereine des collectivités à la définition et au financement des programmes d'alphabétisation. De plus, la valorisation si nécessaire des langues nationales aurait lieu dans un contexte minimisant les appréhensions des partisans des langues exogènes. On voit tout l'intérêt d'une telle démarche pour récupérer les exclus du système scolaire traditionnel. Cette valorisation économique des langues locales apporterait à ces dernières le crédit supplémentaire indispensable pour leur introduction, à terme, dans le système scolaire classique, à titre de confirmation d'un vécu linguistique des populations locales. Ainsi serait atteint l'objectif recherché par l'approche trilingue, et serait traduite dans les faits la recommandation de l'UNESCO.

\section{Universitê de Montréal}

\section{Références}

APPEL, René et Muysken, Pieter (1992). Language Contact and Bilingualism. Londres, Melbourne, Auckland, Edward Arnold.

BAYLON, Christian (1991). Sociolinguistique : société, langue et discours. Paris, Nathan.

BENJAMIN, Jacques (1972). Les Camerounais accidentatox : la minorité dans un État bicommunautaire. Montréal, Presses de l'Université de Montréal.

BIYA, Paul (1987). Interview du président Biya à la télévision camerounaise le 19 février 1987. Yaoundé, Ministère de l'Information et de la Culture, Direction de l'Information et de la Presse.

BOYER, Henri (1991). Langues en confit. Paris, L'Harmattan.

CALVET, Louis-Jean (1987), La guerre des langues. Paris, Payot.

COOPER, Robert L. (1989). Language Planning and Social Change. Cambridge, New York, Port Chester, Melbourne, Sydney; Cambridge University Press. 
DIEU, Michel et Renaud, Patrick (1983). Atlas linguistique du Cameroun (ALCAM) : inventaire préliminaire. Paris, Yaoundé, ACCT, CERDOTOLA, DGRST.

DJTÉ, Paulin G. (1991). "Les langues en Afrique dans la nouvelle francophonie». Language Planning and Language Problems, 15, pp. 20-32.

FERGUSON, Charles A. (1959) «Diglossiaw. Word, 15, pp. 325-340

FISHMAN, Joshua A. (1977). «Language and Ethnicity». In Giles H. (éd.) Language, Ethnicity and Intergroup Relations. Londres, New York, Academic Press, pp. 15-57.

(1967). "Bilingualism With and Without Diglossia ; Diglossia With and Without Bilingualism». Journal of Social Issues, 32, pp. 29-38.

FONLON, Bernard (1975). «The Language Problem in Cameroon : a Historical Perspectiven. In Smock, D. R. et Bentsi-Enchill, K. (éds.). The Search for National Integration in Africa. New York, The Free Press, pp. 189-205.

(1963). «A Case for Early Bilingualism». Abbia, 4, pp. 56-94.

GAILlARD, Philippe (1989). Le Cameroun. (Tome 2) Paris, L'Harmattan.

GARDNER R. C. et Smythe, P.C. (1976). Language Research Group National Test. London, Ontario, University of Western Ontario.

HAMERS, J. F. et Blanc, M. (1992). Bilinguality and Bilingualism. Newcastle, Cambridge University Press.

KGNIG, Edna L., Chia, Emmanuel et Povey, John (1983). A Sociolinguistic Profile of Urban Centers in Cameroon. Los Angeles, Crossroads Press.

LAFONTAINE, Dominique (1986). Le parti-pris des mots : normes et attitudes linguistiques. Bruxelles, Pierre Mardaga. 
MANESSY, Gabriel (resp. scient) (1992). "Normes endogènes et normes pédagogiques en Afrique noire francophonew. In Baggioni, Daniel et al. Multilinguisme et développement dans l'espace francophone. Université de Provence, Didier Érudition, pp. 43-81.

MOPOHO, Raymond (1993). Multilinguisme et communication au Cameroun Études sociolinguistiques. Thèse présentée à la Faculté des études supérieures de l'Université de Montréal en vue de l'obtention du grade de Philosophiæ Doctor (Ph. D.).

NGIJOL, NGIJOL, Pierre (1964). «Nécessité d'une langue nationale». Abbia, 7, pp. 83-99.

RENAUD, Patrick (1987). «Politogenèse et politique linguistique : le cas du Cameroun». Études de linguistique appliquée, 65, nouvelle série, pp. 23-36.

STARK, Frank M. (1980). "Federalism in Cameroon : The Shadow and the Reality. In Kofele-Kale, Ndiva (éd.). An Experiment in Nation Building : The Bilingual Cameroon Republic since Reunification. Boulder, Colorado, Westview Press.

STUMPF, Rudolf (1979). La politique linguistique au Cameroun de 1884 à 1960. Berne, Francfort/Main, Las Vegas, Peter Lang.

TABOURET-KELLER, Andrée (1992).«Some Major Features of the Sociolinguistic Situation in Europe and the European Charterm. In Bolton, Kingsley et Kwok, Helen (eds). Sociolinguistics Today : An International Perspective. Londres et New York, Routledge, pp. 266-280.

TADADJEU, Maurice (1988). «Langue, culture et éducation dans la recherche d'un modèle social". Annales de la Faculté des Sciences humaines de l'Université de Yaoundé, IV, 2, pp. 3-18.

TADADJEU, Maurice (1987). «Le facteur linguistique du projet social camerounais". Journal of West African Languages, 17, pp. 23-34. 
TADADJEU, Maurice (1985). «Pour une politique d'intégration linguistique camerounaise : le trilinguisme extensif". In MINFOC (éd.). L'identité culturelle camerounaise. Yaoundé, Ministère de L'Information et de la Culture, pp. 187-202.

TADADJEU, Maurice (1983). «Prospects for Language Planning Studies in Cameroon». In Kcenig, Edna L., Chia, Emmanuel et Povey, John (eds). A Sociolinguistic Profile of Urban Centers in Cameroon. Los Angeles, Crossroads Press, pp. 117-124.

TODD, Loreto (1983). «Language Options for Education in a Multilingual Society : Cameroon". Kennedy, Chris (éd.). Language Planning and Language Education. Londres, Boston, Sidney; George Allen et Unwin, pp. 160-171.

TOWA, Marcien (1975). «Langue nationale et personnalité nationale». Abbia, 29-30, pp. $95-120$.

VALDMAN, Albert (dir.) (1979). Le français hors de France. Paris, Honoré Champion.

WARDAUGH, Ronald (1987). Languages in Competition. Oxford, Basil Blackwell et New York, and André Deutsch.

YINGER, J. M. (1981). «Toward a Theory of Assimilation and Dissimilation". Ethnic and Racial Studies, 4 (3), pp. 249-264.

RÉSUMÉ : Le bilinguisme officiel (français-anglais) au Cameroun : un problème d'aménagement efficace - Cette étude vise à vérifier l'étendue de la prépondérance exclusive du français et de l'anglais dans la communication au sein de l'État camerounais, en raison de certaines contradictions (Renaud 1979 et 1987). Ces langues officielles sont censées y assurer les fonctions les plus hautes en présence de 236 langues autochtones. Ces dernières sont-elles réellement absentes des services publics? Quelles sont les attitudes des Camerounais envers elles et leurs communautés constitutionnelles respectives? Quelle serait une politique linguistique réaliste pour le Cameroun? 
Pour répondre à ces questions, nous avons, en 1993, soumis à 590 personnes dans les chefs-lieux administratifs des dix provinces du pays, un questionnaire ferme sur leurs choix de langue et les attentes linguistiques perçues par eux dans leurs contacts officiels. L'analyse des données a révélé l'existence d'une concurrence participative reflétant l'élargissement fonctionnel des langues non officielles. Une tension y est apparue entre le français et l'anglais, mais elle reflète les conflits politiques ambiants.

S'agissant des attitudes, le répondant devait porter son jugement sur les langues officielles et leurs communautés constitutionnelles respectives, par rapport à sept différenciateurs sémantiques inspirés de Gardner et Smythe (1976). L'analyse révèle : une haute appréciation des répondants de chaque communauté pour leur première langue officielle, l'évaluation négative de leur deuxième langue officielle par les anglophones, l'apparition des stéréotypes négatifs envers le pidgin, et la meilleure image que les anglophones auraient de leur communauté.

La concurrence participative entre langues officielles et non officielles, le nationalisme et la volonté d'ouverture des Camerounais, ainsi que leur aspiration a voir les hautes fonctions étendues aux langues locales, nous ont amené à proposer l'alphabétisation fonctionnelle en langues locales, pour la nécessaire valorisation des langues endogènes, dans un contexte minimisant la confrontation avec les langues officielles, et consacrant le passage du culturel à l'économique.

\section{ABSTRACT : Official Bilingualism (French-English) in Cameroon :} A Question of Efficient Planning - This study aims at a verification of the extent of exclusive predominant usage of French and English in public offices in Cameroon, in communication within the Cameroonian State, since some contradictions exist (Renaud, 1979 and 1987). The official languages are supposed to assume the highest functions among 236 home languages. Are these actually absent from public services? What are the attitudes of Cameroonians toward these official languages and their respective communities as per the Cameroon Constitution? What would be a realistic language policy for Cameroon?

To answer these questions, a closed survey on language choices and perceived expectations in official contacts was submitted in 1993 to 590 persons in the administrative headquarters of Cameroon's ten provinces. Data analysis showed participative competition as a reflection of a widening of functions assumed by non official languages. Tension 
between French and English was noticed, as a manifestation of local political conflicts.

As concerns attitudes, those surveyed were asked to express their judgment on official languages and their respective communities, according to seven semantic differentials taken from Gardner and Smythe (1976). The analysis revealed : high levels of appreciation by members of each community, of their first official language, a negative evaluation by Anglophones of their second official language, the negative stereotypes of Pidgin English, a better image of their own community as perceived by Anglophones.

On account of the participative competition between official and non official languages, the nationalism and open-mindedness of Cameroonians, as well as their aspiring to a widening of higher linguistic functions to include home languages, functional literacy in local languages is recommended, as a means to solve the necessary enhancement of endogenous languages, with minimal confrontation with official languages, as a consecration of an evolution from the cultural to the economic aspect of language.

Dieudonné P. Aroga Bessong : Direction des services linguistiques, Présidence de la République, YAOUNDÉ, République du Cameroun, Afrique centrale. 